\title{
Improving the Spatial and Energy Resolution of Aberration-Corrected STEM
}

\author{
O.L. Krivanek, N. Dellby, M.F. Murfitt, N.J. Bacon, G.J. Corbin, P. Hrncirik, J.T. Nelson, T.C. \\ Lovejoy, G.S. Skone, and Z.S. Szilagyi
}

Nion Co., $11028^{\text {th }}$ St., Kirkland, WA 98033

Aberration-Corrected Scanning Transmission Electron Microscopes (ACSTEMs) continue to progress and explore new horizons, in several directions at once:

a) Atomic-resolution elemental mapping by EELS [1] and EDXS [2]. Introduced just 3 and 1 years ago, respectively, these techniques have now become standard tools of materials science.

b) Studies of EELS fine structures at atomic resolution and atomic-resolution EELS fine structure mapping [3].

c) Reaching atomic resolution at operating energies lower than $80 \mathrm{keV}$ [4], which is useful for avoiding knock-on damage in low-Z materials such as graphene and monolayer $\mathrm{BN}$, and as low as $30-40 \mathrm{keV}$, which is useful for minimizing the radiation damage at graphene edges.

d) Operating at energies of $200 \mathrm{keV}$ and higher, to reach spatial resolution of $\sim 0.5 \AA$, to optimize penetration of thicker samples, and to minimize ionization damage in some samples.

e) Minimizing sample contamination and etching by providing true UHV ( $10^{-10}$ torr $)$ at the sample.

The above techniques are typically performed in just one "multi-purpose" instrument, and it is therefore fortunate that most of the above requirements are in fact inter-related. Reaching $0.5 \AA$ resolution at $200 \mathrm{keV}$ requires the correction of both $3^{\text {rd }}$ and $5^{\text {th }}$ order aberrations. The resultant high angular range of the probe then allows a large current to be packed into a small probe, typically $\sim 0.5$ $\mathrm{nA}$ into a $1 \AA$ probe at $200 \mathrm{keV}$, which permits rapid mapping. The limit on the spatial resolution in such an instrument comes mainly from chromatic aberration, which means that the chromatic aberration coefficient of the probe-forming column and the energy spread of the electron source need to be as small as possible. A cold field emission gun (CFEG), which requires hard UHV, has an energy spread of $\sim 0.3 \mathrm{eV}$, and this gives it a major advantage over Schottky guns with energy spread of $\sim 0.6 \mathrm{eV}$. The $0.3 \mathrm{eV}$ energy spread of the CFEG is also helpful for good EELS energy resolution.

Figs 1-3 illustrate the performance of modern CFEG ACSTEMs with examples from the Nion UltraSTEM200 at CNRS Orsay, France. Fig. 1 shows HAADF information transfer to $(0.53 \AA)^{-1}$ at $200 \mathrm{keV}$, on a Au particle. Fig. 2 shows that the same microscope can resolve $1.2 \AA$ spacings at 40 $\mathrm{keV}$. Fig. 3 shows an EELS zero loss peak $0.26 \mathrm{eV}$ wide obtained at $100 \mathrm{keV}$.

To progress beyond $0.25 \mathrm{eV}$ energy resolution and $1 \AA$ spatial resolution at $60 \mathrm{keV}$, new approaches are needed. For substantially better energy resolution, monochromation is essential. The narrower energy spread should then lead to better STEM spatial resolution. This has not yet been shown experimentally, probably because monochromation produces a significant decrease of the beam current, and compensating for the decrease can lead to major probe broadening due to the finite brightness of the source. Minimizing this effect requires extra-bright electron guns and dispersingundispersing monochromators, in which the energy dispersion that's needed at the energy-selecting slit is precisely canceled before the beam is sent into the rest of the electron-optical column.

Fig. 4 shows the cross-section through a Nion UltraSTEM column that includes a dispersingundispersing monochromator that we are now building. The monochromator provides a way to improve the energy stability of the total system by linking the energy of the beam selected by the slit 
to the energy analyzed by the EEL spectrometer [5]. It should also be able to correct chromatic aberration, thus potentially providing an improved spatial resolution without a substantial loss of beam current. When it is fully working, it should be able to reach spatial resolution of $0.5 \AA$ at 100 $\mathrm{keV}$ primary energies and below, and an energy resolution better than $30 \mathrm{meV}$.

We are grateful to all our collaborators for support, and to the Orsay STEM group, especially to Dr. C. Colliex, for their contributions to the development of the Nion $200 \mathrm{keV}$ ACSTEM.

References

[1] D.A. Muller et al., Science 319, 1073-1076 (2008).

[2] A.J. D’Alfonso et al., Phys Rev. B81 100101(R) (2010).

[3] L.F. Kourkoutis et al., PNAS 90, $4731-4749$ (2010).

[4] O.L. Krivanek et al., Ultramicroscopy 110, 935-945 (2010).

[5] O.L. Krivanek et al., Phil. Trans. R. Soc. A 2009 367, 3683-3697 (2009).
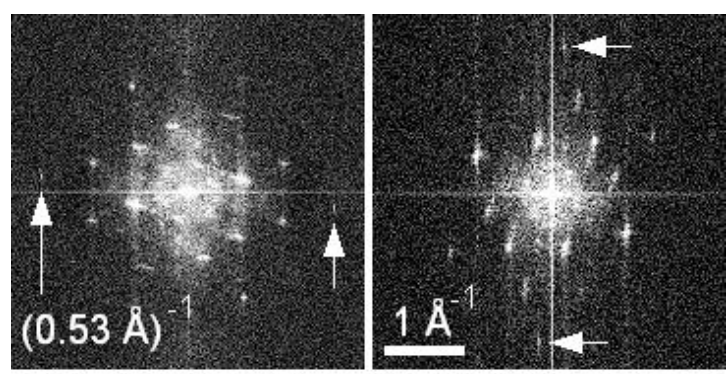

Fig. 1. FFTs of two HAADF images of the same Au particle recorded at $200 \mathrm{keV}$. The scan direction was rotated $90^{\circ}$ for the second image. Arrows point to spots due to planes spaced $0.53 \AA$ apart.

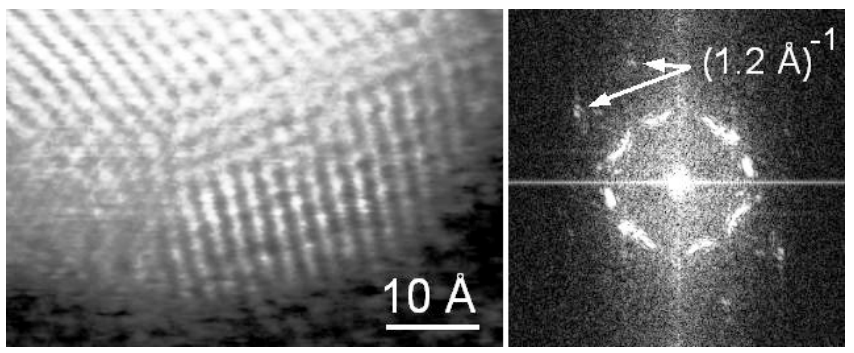

Fig. 2. Part of an HAADF image of Au particle recorded using the "second zone" objective lens mode at $40 \mathrm{keV}$ with $60 \mathrm{pA}$ probe current, and FFT.

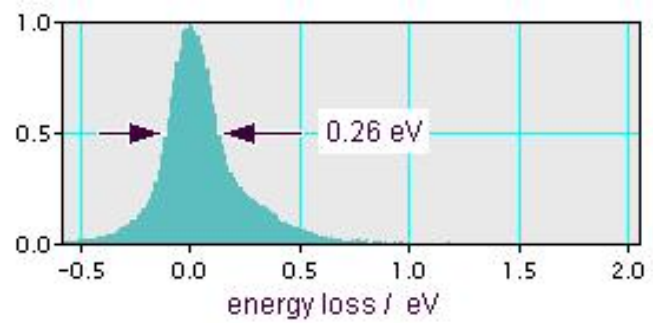

Fig. 3. Zero Loss EELS peak, $0.26 \mathrm{eV}$ wide (FWHM), recorded at $100 \mathrm{keV}$.

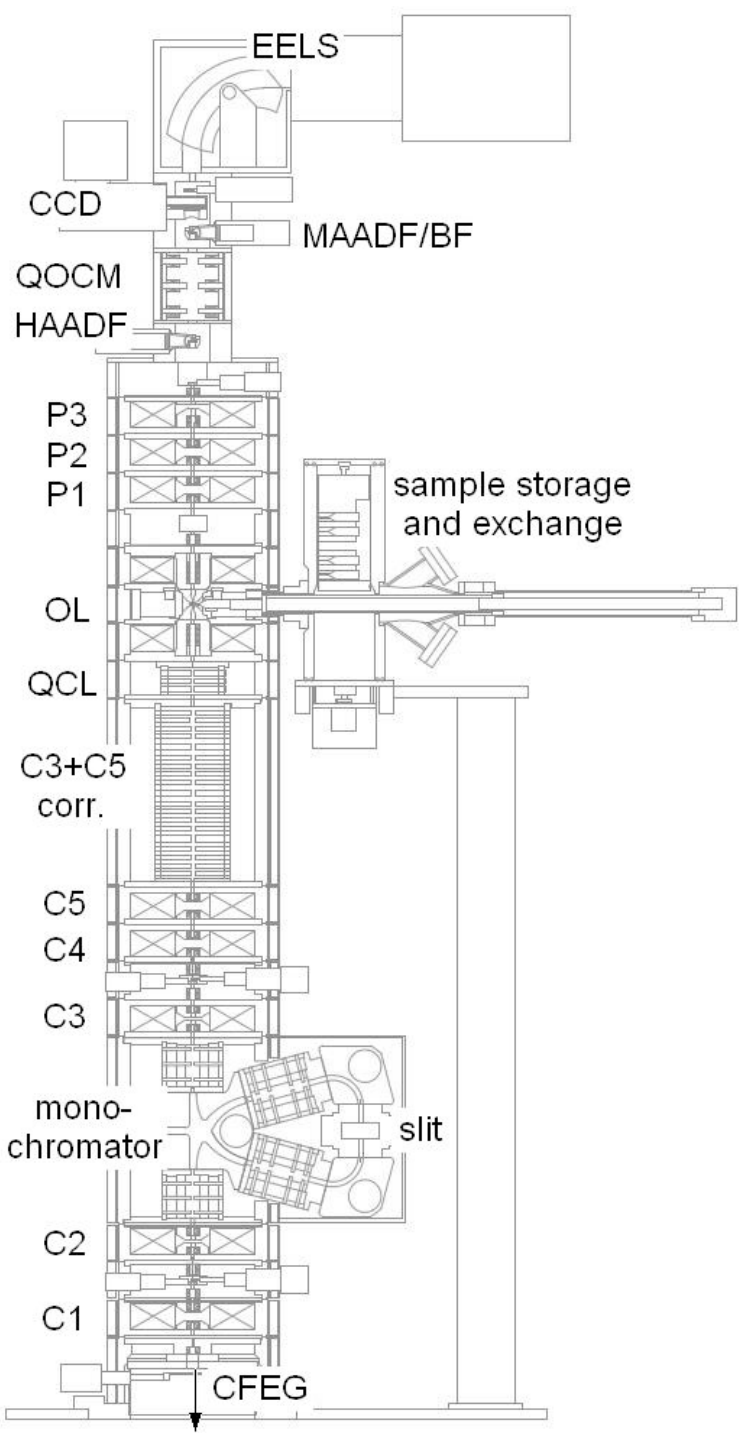

Fig. 4. Cross-section of Nion's monochromated CFEG ACSTEM. 